\title{
Review of the Linogram and Sinogram: An Easy Way to Detect Off-Peak Artifacts in Myocardial Perfusion SPECT
}

\author{
Mehdi Mommennezhad, Seyed Rasoul Zakavi, Ramin Sadeghi, and Vahid Reza Dabbagh Kakhki \\ Department of Nuclear Medicine, Emam Reza Hospital, Mashhad University of Medical Sciences, Mashhad, Iran
}

\begin{abstract}
The correct energy window setting and a stable photopeak position are crucial for good data acquisition. We report a patient whose myocardial perfusion SPECT images showed mildly irregular radiotracer uptake when the patient was prone but not when the patient was supine. Review of the sinogram showed clear interruptions in the sinusoidal shape of the raw data when the patient was prone. Detailed technical evaluation revealed that the artifact was caused by an unstable photopeak position due to malfunction of a power supply board of the $\gamma$-camera. This case emphasizes the importance of reviewing the sinogram display and raw data to assess the integrity of data acquisition and to reveal any potential technical problems such as a spontaneous shift of the photopeak.
\end{abstract}

Key Words: sinogram; artifact; myocardial perfusion SPECT; photopeak

J Nucl Med Technol 2009; 37:188-190

DOI: $10.2967 /$ jnmt.108.061218

\section{$\mathbf{M}$} yocardial perfusion SPECT is one of the most common imaging techniques performed in nuclear medicine departments $(1,2)$. Quality control of the $\gamma$-camera is essential to produce high-quality images. Usually, uniformity and linearity correction and calibration are done for each system at periodic intervals. One of the most important tests that should be performed daily is energy peaking (3). It is imperative to visually check the energy window in the spectral display mode with the radionuclide to be imaged before data acquisition. However, unexpected or transient faults in $\gamma$-camera function may result in image artifacts that should be detected and corrected accordingly. Usually, raw data are reviewed for quality of images and patient motion and then images are processed and a snapshot is made as a final print. However, including an image of the sinogram or

Received Dec. 12, 2008; revision accepted May 19, 2009.

For correspondence or reprints contact: Seyed Rasoul Zakavi, Department of Nuclear Medicine, Emam Reza Hospital, Mashhad University of Medical Sciences, Ebne Sina St., Mashhad, Islamic Republic of Iran 9137913316.

E-mail: zakavir@mums.ac.ir

COPYRIGHT @ 2009 by the Society of Nuclear Medicine, Inc. linogram in the final print is an easy way to provide important information regarding not only patient motion but also offpeak artifacts. We report a case of myocardial perfusion SPECT in which interruptions in the linogram and sinogram were due to an unstable photopeak position.

\section{CASE REPORT}

A 45-y-old woman was referred to our department for myocardial perfusion SPECT because of suspected coronary artery disease. Dipyridamole was infused for $4 \mathrm{~min}$ at a dose of $0.568 \mathrm{mg} / \mathrm{kg}$ of body weight, and $740 \mathrm{MBq}$ of 99mTc-methoxyisobutylisonitrile were injected 4 min later. Myocardial perfusion SPECT was done with the patient supine $1 \mathrm{~h}$ after injection of the radiotracer. The imaging was performed with a single-head $\gamma$-camera (DSX1; SMV) equipped with a low-energy, general-purpose collimator. Projections were acquired in 32 steps around a $180^{\circ}$ arc for $25 \mathrm{~s}$ per projection starting with the right anterior oblique view and ending with the left posterior oblique view. The total acquisition time was $15 \mathrm{~min}$. The acquisition was in step-and-shoot mode with a zoom factor of 1.23 , and the images were recorded in a $64 \times 64$ matrix. Routinely, we perform stress myocardial perfusion SPECT with the patient supine, the images are reviewed by the physician, and in cases of suspected diaphragmatic attenuation, imaging is repeated immediately with the patient prone.

In this patient, supine SPECT images showed slightly decreased uptake in the inferior wall, and prone imaging was requested. The prone view, which was obtained immediately after supine imaging, showed mild irregularity of uptake throughout the myocardium (Fig. 1). In this patient, the sinogram and linogram of the prone images showed multiple interruptions suggesting low-count images (Fig. 2) in some projections. The semisinus form of the sinogram was preserved, indicating that no motion had occurred, but the counting rate was significantly decreased in some projections. Figure 2 showed clear interruptions in the shape of the sinogram and linogram on prone images but a normal shape on supine images. Raw data were reviewed for the total counts and duration of each projection (Fig. 3) and showed lower counts in many projections, especially in the first two 


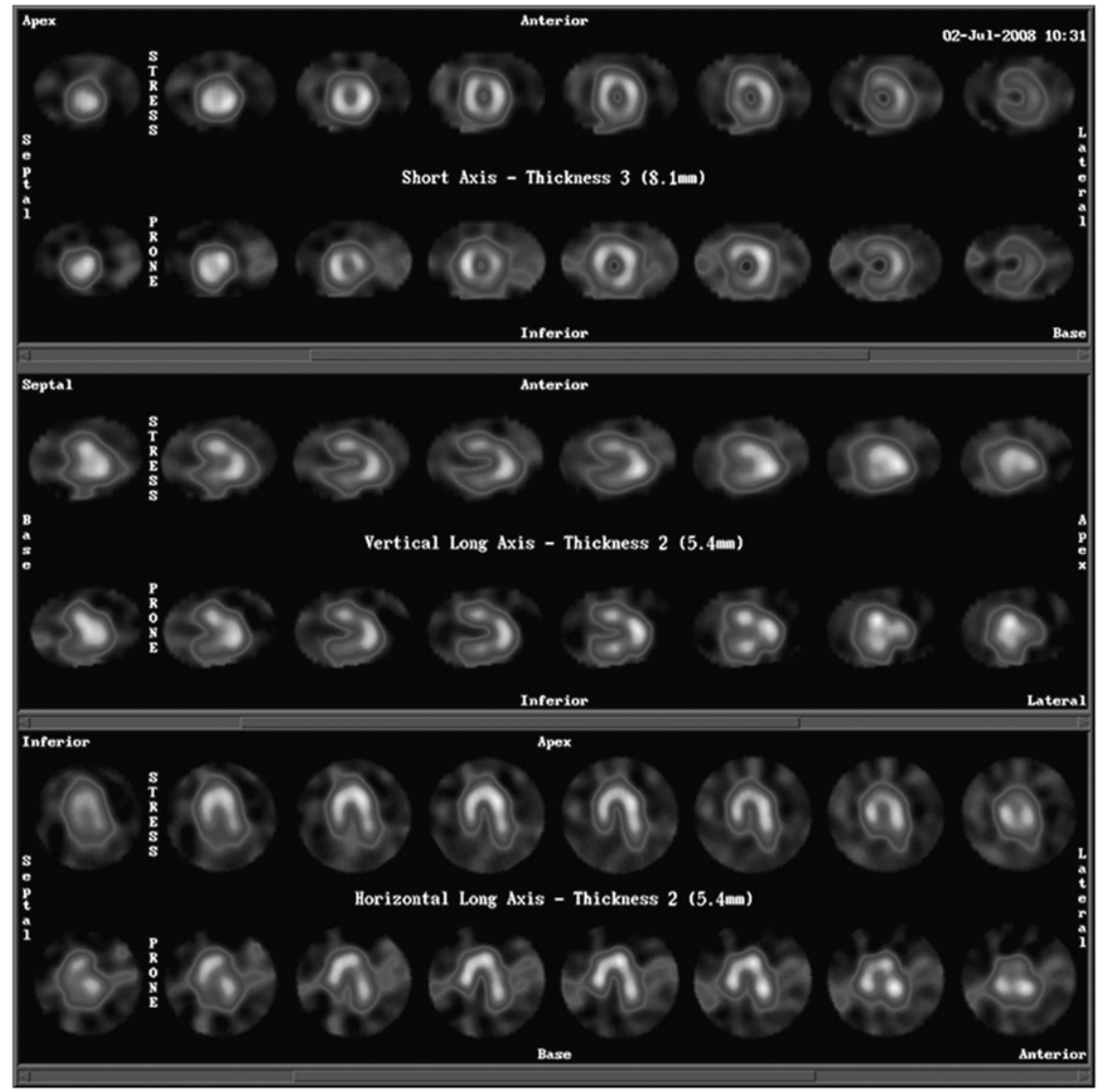

FIGURE 1. Supine (upper row) and prone (lower row) myocardial perfusion SPECT. Note irregularity of radiotracer uptake in myocardial walls on prone images. thirds of images, corresponding to the interruptions in the sinograms and linograms. We tested the camera using a sample of radioactivity and monitored the photopeak position with respect to the energy window during acquisition. The test showed that the photopeak shifted in several images beyond the determined energy window. The camera was checked by service engineers; a loose connection in the electronic board of the power supply was detected, and the board was replaced. Repeated testing showed that the photopeak position was stable.

\section{DISCUSSION}

It is reported that an incorrect energy window setting, an unstable energy window, and spontaneous shifting of the peak may cause artifacts in reconstructed images (4-6). Although energy window settings are usually checked every day in clinical practice, spontaneous shifting in the photopeak or an unstable energy window may not be detected in routine quality control tests. However, such changes may affect the quality of images significantly (6).

Sinograms and linograms may be reviewed by the operator as an index for motion artifacts (7). The semisinus shape of the sinogram suggests an absence of motion artifacts, but because the count of each projection is also reflected in each row of the sinogram, the discontinuity of the sinogram can be used as an index for count loss in different projections.
The loss in the recorded counts may be due to a spontaneous shift of the photopeak position with respect to the energy window and may affect the quality of the raw

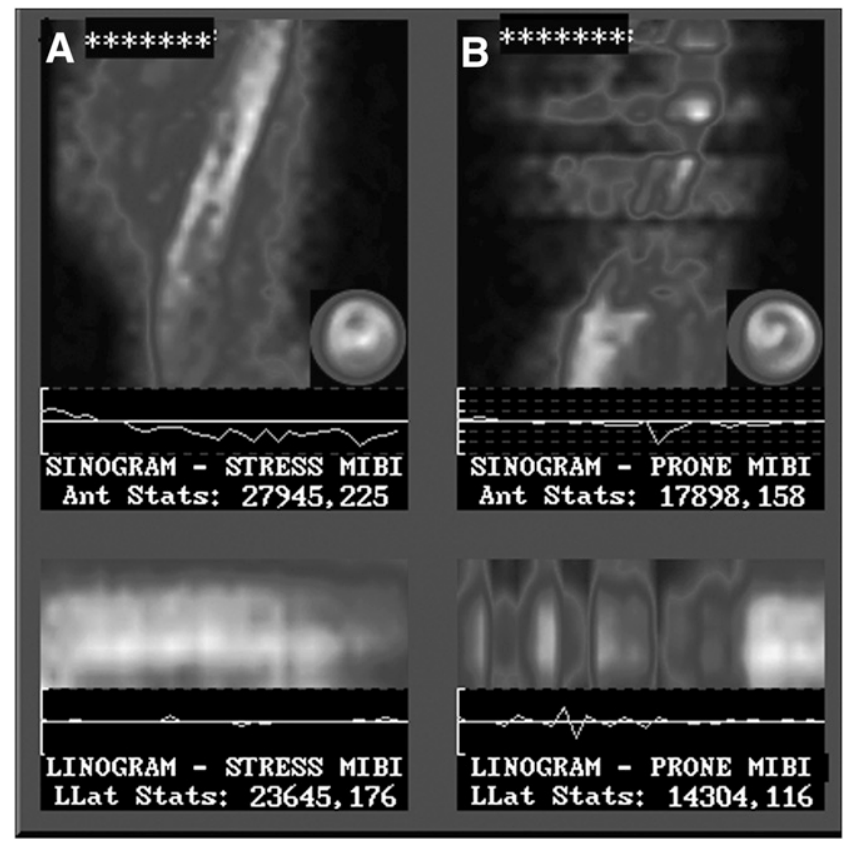

FIGURE 2. Linogram and sinogram of supine $(A)$ and prone $(B)$ myocardial perfusion SPECT. Note interruptions in linogram and sinogram on prone images. 


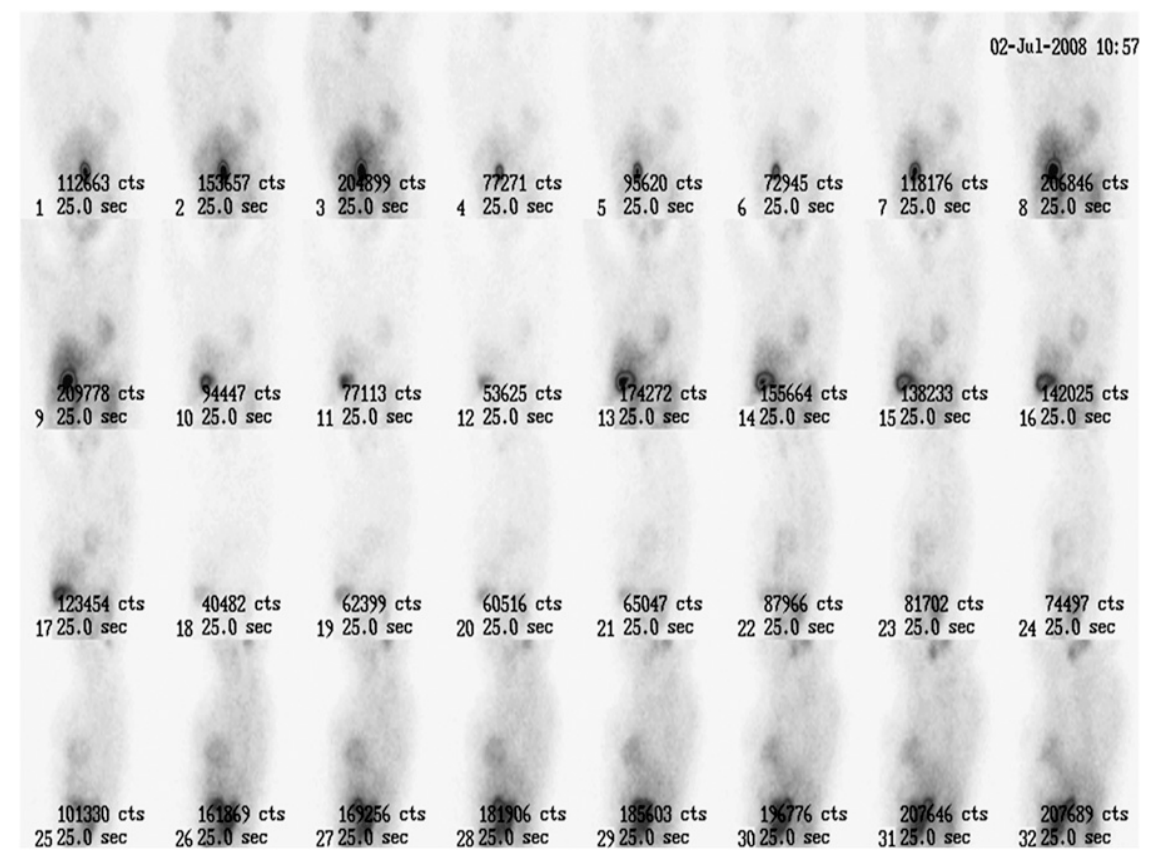

FIGURE 3. Thirty-two projections, $25 \mathrm{~s}$ each, obtained during myocardial perfusion SPECT. Total counts and intensity of images are significantly different among projections.

data. It seems that the degraded images would be easily detectable in dynamic planar studies as a result of poor counts or information density in images. However, count loss during SPECT imaging can cause artifacts in the reconstructed images and may result in false scoring of the segments in semiquantitative analysis. These artifacts are difficult or impossible to recognize without reviewing the sinogram or linogram and may lead to an incorrect interpretation. In addition, review of the raw images in cine mode may show a flip-flop or flickering pattern dependent on the frequency of the images that have count loss. It was also reported that review of raw data and sinograms is essential to ascertain the integrity of the SPECT projection data (8). However, that reported study was done with a triple-head camera using $360^{\circ}$ of acquisition, which is not commonly used for myocardial perfusion SPECT (8). We report a case of myocardial perfusion SPECT that was done with a single-head camera using a $180^{\circ}$ arc.

It becomes more important that the physician be able to recognize the various types of image artifacts that may occur due to spontaneous $\gamma$-camera malfunction. This case review introduces an unusual but important artifact caused by spontaneous $\gamma$-camera malfunction. We conclude that including a sinogram image in the final print of the myocardial perfusion SPECT examination may be helpful in the detection of not only motion artifacts but also a transient photopeak or window shift. In our $\gamma$-camera, the shift in the photopeak position was caused by a malfunctioning power supply board. The problem was solved by replacing the power supply board with a new one.

\section{REFERENCES}

1. Koizumi K, Tamaki N, Inoue T, et al. Nuclear medicine practice in Japan: a report of the 5th nationwide survey in 2002. Ann Nucl Med. 2004;18:73-78.

2. Zakavi SR. Nuclear cardiology in Iran. Iran J Nucl Med. 2002;21:73-77.

3. Zanzonico P. Routine quality control of clinical nuclear medicine instrumentation: a brief review. J Nucl Med. 2008;49:1114-1131.

4. Shih WJ, Schoenstra T, Gross K, Wierzbinski B, Kiefer V, Collins J. Electronic off-peak status of one head of a dual-head gamma camera resulted in bone scintigraphy artifacts and faulty finding on gated myocardial SPECT. $J$ Nucl Med Technol. 2003;31:165-169.

5. Al-Lehyani SHA. Effect of peak shift on planar and SPECT images. J King Saud Univ. 2006;19:59-71.

6. Nitke SJ. Off-peak detector mimicking ischemia on myocardial scan. Clin Nucl Med. 1999;24:276.

7. Lu W, Mackie TR. Tomographic motion detection and correction directly in sinogram space. Phys Med Biol. 2002;47:1267-1284.

8. Busemann Sokole E. IAEA quality control atlas for scintillation camera systems. Vienna, Austria: International Atomic Energy Agency; 2003:222. 